\title{
Plasmonics Based Harsh Environment Compatible Chemical Sensors
}

\author{
Final Report \\ Reporting Period Start Date: 1/16/2009 \\ Reporting Period End Date: 12/31/2011 \\ Principal Author: Dr. Michael A. Carpenter \\ Date Report Issued: April 2012 \\ DOE Award Number: DE-NT0007918 \\ College of Nanoscale Science and Engineering \\ University at Albany-SUNY \\ Albany, NY 12203 \\ Phone: 518-437-8667 \\ Fax: 518-437-8603 \\ Email: mcarpenter@albany.edu
}




\section{Disclaimer}

"This report was prepared as an account of work sponsored by an agency of the United States Government. Neither the United States Government nor any agency thereof, nor any of their employees, makes any warranty, express or implied, or assumes any legal liability or responsibility for the accuracy, completeness, or usefulness of any information, apparatus, product, or process disclosed, or represents that its use would not infringe privately owned rights. Reference herein to any specific commercial product, process, or service by trade name, trademark, manufacturer, or otherwise does not necessarily constitute or imply its endorsement, recommendation, or favoring by the United States Government or any agency thereof. The views and opinions of authors expressed herein do not necessarily state or reflect those of the United States Government or any agency thereof." 


\begin{abstract}
$\mathrm{Au}-\mathrm{YSZ}, \mathrm{Au}-\mathrm{TiO}_{2}$ and $\mathrm{Au}-\mathrm{CeO}_{2}$ nanocomposite films have been investigated as a potential sensing element for high-temperature plasmonic sensing of $\mathrm{H}_{2}, \mathrm{CO}$, and $\mathrm{NO}_{2}$ in an oxygen containing environment. The $\mathrm{Au}-\mathrm{YSZ}$ and $\mathrm{Au}-\mathrm{TiO}_{2}$ films were deposited using PVD methods, while the $\mathrm{CeO}_{2}$ thin film was deposited by molecular beam epitaxy (MBE) and Au was implanted into the as-grown film at an elevated temperature followed by high temperature annealing to form well-defined Au nanoclusters. Each of the films were characterized by x-ray diffraction (XRD) and Rutherford backscattering spectrometry (RBS). For the gas sensing experiments, separate exposures to varying concentrations of $\mathrm{H}_{2}, \mathrm{CO}$, and $\mathrm{NO}_{2}$ were performed at a temperature of $500^{\circ} \mathrm{C}$ in oxygen backgrounds of 5.0, 10, and $\sim 21 \% \mathrm{O}_{2}$. Changes in the localized surface plasmon resonance (LSPR) absorption peak were monitored during gas exposures and are believed to be the result of oxidation-reduction processes that fill or create oxygen vacancies in the respective metal oxides. This process affects the LSPR peak position either by charge exchange with the Au nanoparticles or by changes in the dielectric constant surrounding the particles. Hyperspectral multivariate analysis was used to gauge the inherent selectivity of the film between the separate analytes. From principal component analysis (PCA), unique and identifiable responses were seen for each of the analytes. Linear discriminant analysis (LDA) was also used on the $\mathrm{Au}-\mathrm{CeO}_{2}$ results and showed separation between analytes as well as trends in gas concentration. Results indicate that each of the films are is selective towards $\mathrm{O}_{2}$, $\mathrm{H}_{2}, \mathrm{CO}$, and $\mathrm{NO}_{2}$ in separate exposures. However, when the films were analyzed in a sensor array based experiment, ie simultaneous exposures to the target gases, PCA analysis of the combined response showed an even greater selective character towards the target gases. Combined with the observed stability over long exposure periods, each of these Au-metal oxide films shows good potential as an optical sensing element for harsh environmental conditions.
\end{abstract}




\section{Table of Contents}

Disclaimer

$\begin{array}{ll}\text { Abstract } & \text { iii }\end{array}$

1.0 Executive Summary 2

2.0 Disseminated Results 3

3.0 Experimental Methods $\quad 4$

4.0 Sensing Studies

4.1 Parallel Sensing Studies 6

4.2 Au nanoparticle Size Dependent Studies 7

4.3 Kinetics Study 9

4.4 Selectivity and Material Control : Au-ceria Sensing Results $\quad 10$

4.5 Array Data: PCA Analysis 13

4.6 Material Control : Embedded Gold Nanorods 14

$\begin{array}{ll}5.0 \text { Conclusions } & 16\end{array}$

$\begin{array}{lr}6.0 \text { Acronyms } & 16\end{array}$

$\begin{array}{ll}7.0 \text { References } & 18\end{array}$ 


\subsection{Executive Summary}

The work pursued during this program focused on five inter-related initiatives which include 1)Parallel Materials Deposition, 2)Parallel Sensing Studies, 3) Sensing Characterization, 4)Materials Optimization, and 5)Selectivity Studies. These five initiatives are critical towards our goal of developing the plasmonics based harsh environment compatible chemical sensors. As with any sensor development program we are aiming to detect with a high degree of sensitivity and selectivity our target emission gases. The approach we took was to develop expertise in the parallel deposition of nanocomposite materials, these materials are then interrogated in a parallel sensing testing station which has been optimized to the point where we are challenged in our deposition and sample processing methods moreso than our sample imaging capabilities. Analysis of the equilibrium and kinetically limited data has led to a deeper understanding of the surface and core reactions which govern the observed $\mathrm{Au}$ nanoparticle sensor response. Metal oxides used in this study for encapsulation of the Au nanoparticles included yttria stabilized zirconia, ceria and titania. A series of plasmonic sensor characterization studies were performed on these nanocomposite films to determine their efficacy for the detection of $\mathrm{H}_{2}, \mathrm{CO}$ and $\mathrm{NO}_{2}$ in an air or oxygen containing carrier gas and operating temperatures of $500^{\circ} \mathrm{C}$. The nanocomposites showed reliability over time periods of approximately 100-150 hours with a detection limit of $\mathrm{NO}_{2}$ at $2 \mathrm{ppm}$. Principal component analysis was used as a statistical algorithm to characterize the selectivity of the sensing arrays response and an array containing $\mathrm{Au}-\mathrm{YSZ}, \mathrm{Au}-\mathrm{TiO}_{2}$ and $\mathrm{Au}-\mathrm{CeO}_{2}$ showed a unique fingerprint response to the target gases under harsh operating conditions. Two Ph.D. graduate students have worked on this project, one graduated in winter 2009 and the $2^{\text {nd }}$ one has approximately a year left before he has completed his degree. Three journal articles, 3 conference proceedings, a book chapter and a book which the PI has edited have been completed during this program and 17 conference presentations or invited presentations have been given within the period of this contract. 


\subsection{Disseminated Results Publications}

1. M. A. Carpenter, "Optical Sensing Methods for Metal Oxide Nanomaterials", Metal Oxide Nanomaterials for Chemical Sensors, Springer (to be published 2012)

2. Metal Oxide Nanomaterials for Chemical Sensors, Editors: M.A. Carpenter, S. Mathur, A. Kolmakov, Springer (to be published 2012)

3. N. A. Joy, P. H. Rogers, M. A. Carpenter, "Principal Component Analysis of $\mathrm{Au}-\mathrm{CeO}_{2}$ Plasmonic Sensing Response to $\mathrm{H}_{2}, \mathrm{NO}_{2}$, and CO", Analyt. Chem., 84, 5025 (2012).

4. Joy, N. A., Settens, C. M., Matyi, R J., Carpenter, M. A., "Plasmonic Based Kinetic Analysis of Hydrogen Reactions within Au-YSZ Nanocomposites", Journal of Physical Chemistry C 115, 6283-6289, (2011).

5. P. H. Rogers, M. A. Carpenter, "Particle Size Sensitivity Dependence of Nanocomposites for Plasmonic-Based All-Optical Sensing Applications", Journal of Physical Chemistry C, 114, 11033 (2010).

6. P. H. Rogers, N. Joy, M. A. Carpenter, "Plasmonic Based Harsh Environment Compatible Chemical Sensors" Proceedings of the $4^{\text {th }}$ International Conference on Sensing Technology, (June 2010)

7. P. H. Rogers and M. A. Carpenter, "Defect State Dampening of Surface Plasmons in Au-YSZ Nanocomposites", Proceedings of the SPIE (2009 Fall Meeting)

8. P. H. Rogers and M. A. Carpenter, "Characterization of Surface Plasmon Peak Shifts and Dampening in Au-YSZ Nanocomposites", Proceedings of the SPIE (2009 Fall Meeting)

\section{Presentations}

1. M. A. Carpenter, "Plasmonic Based Harsh Environment Compatible Chemical Sensors", Electrical and Computer Engineering Department, University of Minnesota-Twin Cities, (November 2011), Invited

2. M. A. Carpenter, "Plasmonic Based Harsh Environment Compatible Chemical Sensors", $7^{\text {th }}$ Annual Minnesota Nanotechnology Workshop, University of Minnesota-Twin Cites, (November 2011), Invited

3. M. A. Carpenter, "Plasmonic Based Harsh Environment Compatible Chemical Sensors", Composites at Lake Louise, Calgary, Alberta, (November 2011), Invited

4. N. A. Joy, M. Nandasiri, T. Varga, V. Shutthanandan, W. Jiang, P. Nachimuthu, S. Kuchibhatla, S. Thevuthasan, M. A. Carpenter' "Harsh Environment Plasmonic Sensing Using an Array of Gold-Metal Oxide Nanocomposite Films", Materials Research Society (November 2011).

5. N. A. Joy, M. Nandasiri, T. Varga, V. Shutthanandan, W. Jiang, P. Nachimuthu, S. Kuchibhatla, S. Thevuthasan, M. A. Carpenter, "Growth and Characterization of Auimplanted MBE Grown $\mathrm{CeO}_{2}$ Thin Films for Plasmonic Based Chemical Sensors", American Vacuum Society (November 2011).

6. M. A. Carpenter, "Plasmonic Based Harsh Environment Compatible Chemical Sensors", Department of Materials Science and Engineering, Stony Brook University-SUNY, (September 2011), Invited

7. M. A. Carpenter, "Plasmonic Based Harsh Environment Compatible Chemical Sensors", Goodrich Inc., (March 2011), Invited

8. M. A. Carpenter, "Plasmonic Based Harsh Environment Compatible Chemical Sensors", Department of Chemistry, The Ohio State University, (March 2011), Invited 
9. M. A. Carpenter, "Plasmonic Based Harsh Environment Compatible Chemical Sensors", $12^{\text {th }}$ International Ceramics Congress, CIMTEC 2010, Montecatini Terme, Italy, (June 2010), Invited

10. M. A. Carpenter, "Plasmonic Based Harsh Environment Compatible Chemical Sensors", $4^{\text {th }}$ International Conference on Sensing Technology, ICST 2010, Lecce, Italy, (June 2010)

11. M. A. Carpenter, "Plasmonic Based Harsh Environment Compatible Chemical Sensors", American Chemical Society, (March 2010), Invited

12. P. H. Rogers, N. Joy, M. A. Carpenter, "Plasmonic-based Detection of Harsh Environment Emissions Gases by Thin Film Nanocomposites", Materials Research Society, (December 2009)

13. P. H. Rogers, N. Joy, M. A. Carpenter, "Characterization of Charge Exchange and Oxygen Ion Formation by Localized Surface Plasmon Resonance Shifts in Au YttriaStabilized Zirconia Nanocomposites", Materials Research Society, (December 2009)

14. M. A. Carpenter, "Plasmonics Based Harsh Environment Compatible Chemical Sensors", PACRIMS 8, American Ceramics Society Meeting, June 2009, Invited

15. M. A. Carpenter, "Plasmonics Based Harsh Environment Compatible Electrochemical Sensors", National Nanotechnology Initiative -Nanotechnology Enabled Sensing Workshop, May 2009, Invited

16. P. H. Rogers, M. A. Carpenter, "Defect State Dampening of the Au Nanoparticle SPR Band in Au-YSZ Nanocomposites in Harsh Environments", $33^{\text {rd }}$ International Conference on Advanced Ceramics and Composites, American Ceramics Society, (January 2009).

17. P. H. Rogers, M. A. Carpenter, "Development and Study of Au- $\left(\mathrm{Y}_{2} \mathrm{O}_{3}\right)_{\mathrm{x}}\left(\mathrm{ZrO}_{2}\right)_{\mathrm{y}}$ Nanocomposites Films for All-Optical Harsh Environment Chemical Sensing Applications", $33^{\text {rd }}$ International Conference on Advanced Ceramics and Composites, American Ceramics Society, (January 2009). -Invited

\subsection{Experimental Methods}

Au-YSZ thin films have been deposited using rf co-magnetron confocal physical vapor deposition (PVD) from a $\mathrm{Au}$ target (99.99\% purity) and a $5 \mathrm{wt} \% \mathrm{Y}_{2} \mathrm{O}_{3}-\mathrm{ZrO}_{2}$ target $(99.9 \%$ purity), purchased from Williams Advance Materials (Brewster, NY). Shutters obstructing the substrates from the targets were opened after 15 minutes of stabilization at deposition power and the films were deposited in 5 minutes on sapphire substrates in a 5 mTorr Ar environment. Depositions took place with the Au target at $20 \mathrm{~W}$ and the YSZ target at $200 \mathrm{~W}$ to ensure a $\sim 10$ at. \% Au composition in the nanocomposite film. ${ }^{1,2}$ After deposition, films were removed from the deposition chamber and placed into an annealing chamber. The films were then annealed at $\sim 900{ }^{\circ} \mathrm{C}$ for 2 hours under $\sim 1800 \mathrm{sccm}$ of Ar at atmospheric pressure. Film thicknesses of the deposited films were $\sim 30 \mathrm{~nm}$ as measured using scanning electron microscopy.

After annealing and characterization, the Au-YSZ films are placed in a Macor sample holder centered in an optically transparent quartz flow cell housed in a tube furnace. Collimated light is transmitted through the film and is analyzed using an Oriel Instruments (Stratford, CT) MS257 monochromator equipped with a Peltier cooled CCD. As schematically depicted in Figure 2, the gas exposure bench schematic shows an expanded view of the Au-YSZ film deposited on the sapphire substrate. This expanded view shows only half of the $1 \mathrm{~cm}$ diameter sapphire substrate is coated with a Au-YSZ film. This film coverage is achieved through the use of either a shadow 
mask during the PVD process or by assembling individual samples into the Macor holder and with the addition of an uncoated sample online background data correction can be performed on every spectrum acquired by the monochromator, resulting in more accurate absorption data acquired for the Au-YSZ thin films. The total gas flow was held at $2000 \mathrm{sccm}$ with gas mixtures controlled by MKS (Wilmington, MA) mass flow controllers attached to a stainless steel gas flow manifold coupled to the quartz flow cell inlet. A schematic of this system is shown in Figure 1.

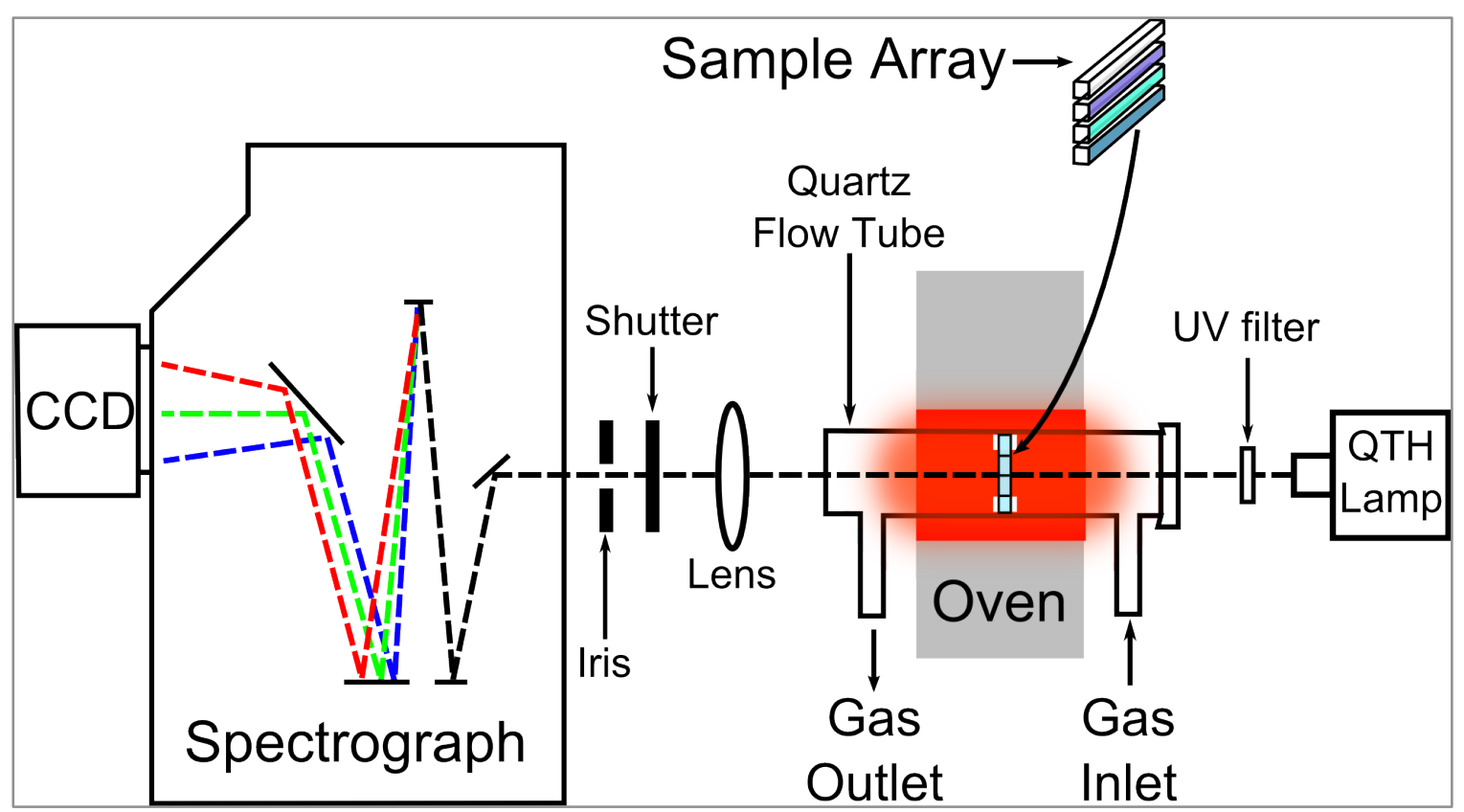

Figure 1: Schematic of experimental apparatus used for acquisition of plasmonic absorption spectra as a function of temperature and gas exposure. 


\subsection{Sensing Studies}

\subsection{Parallel Sensing Studies}

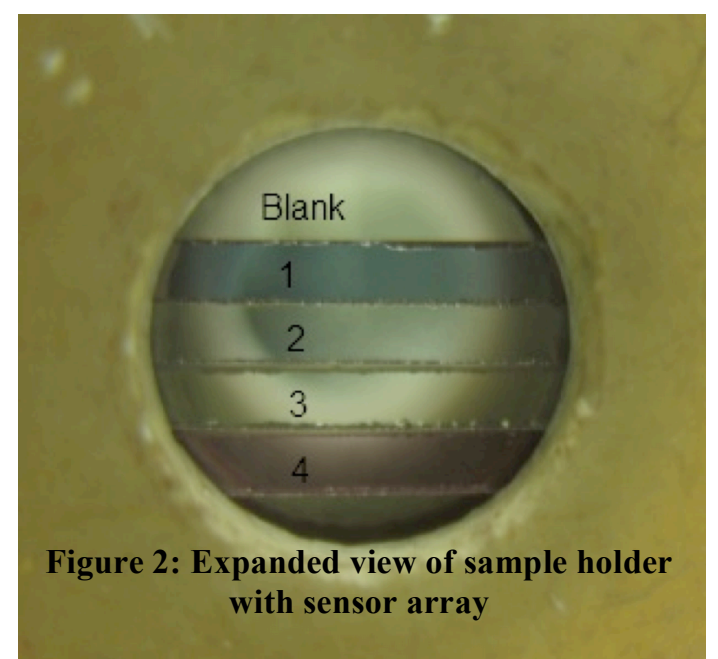

which is useful for optimizing data collection. With this improvement we can in theory image array elements that are limited by the CCD pixel size, the speed data acquisition electronics, and the optical density of the imaged sample element. Currently our main limitation is with how small we can physically cut (or mask) our substrates and still be able to mount them in the Macor sample holder. Currently we can image up to 6 array elements. If more array elements are needed we may in the future explore other means at depositing the array elements, ie a graded deposition scheme. An example of an array comprised of 4 elements is seen in Figure 2.

As a test of this improvement we performed a series of sensor array measurements. Two new sensing films have been made using $\mathrm{TiO}_{2}$ as the metal oxide matrix. These have been grouped with a typical Au-YSZ film and a Au$\mathrm{CeO}_{2}$ sample obtained from Pacific Northwest National Laboratory in order to perform a parallel sensing experiment using different materials rather than variations of the same material. This is the most number of samples we have tested so far in the parallel sensing setup and improvements can still be made to allow for the addition of
To enable an optimized array measurement configuration, time was spent in optimizing the imaging properties of the CCD spectrometer and its corresponding optics. Instead of obtaining an image using collimated light, a lens is now used to project an image of the samples onto the entrance of the imaging components and the quartz flow tube are not preserved as they were with collimated light. Instead, only the sample itself is in focus on the entrance of the spectrometer, resulting in a higher quality image. The use of a lens also has the benefit of being able to magnify or de-magnify the image of the sample

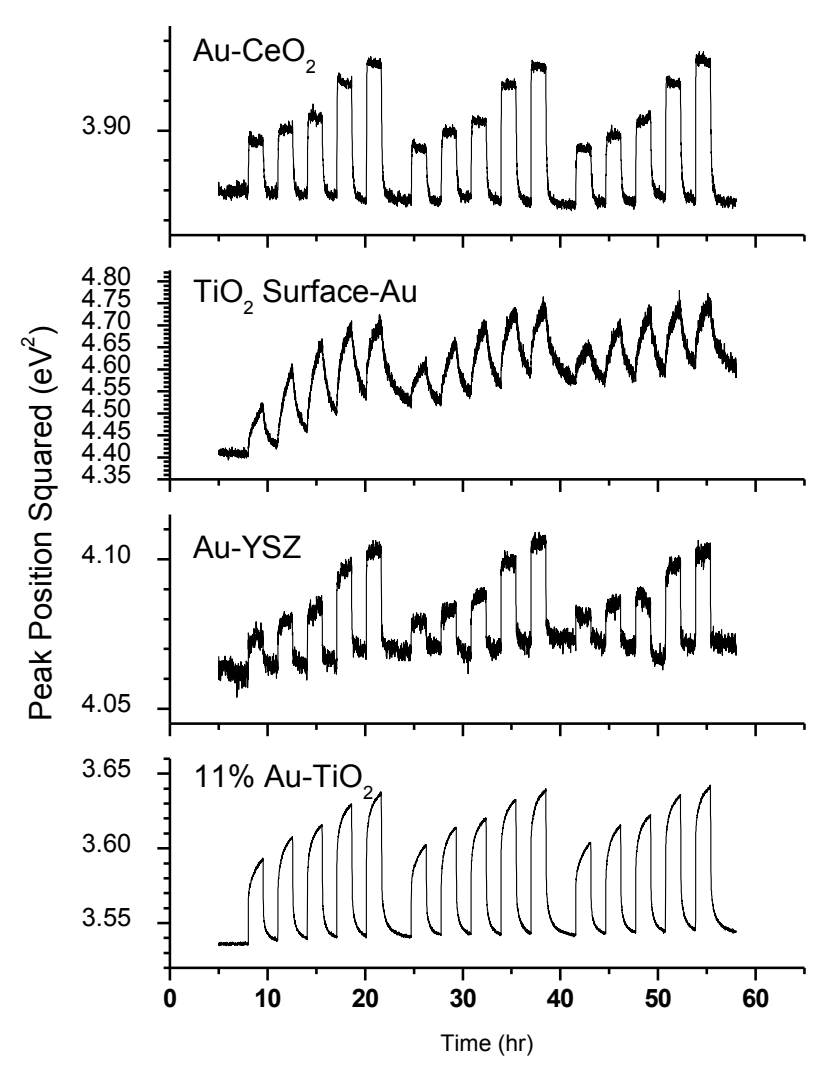

Figure 3: Exposure plots of the four different array elements to the same $\mathrm{H}_{2}$ gas exposures in an air background at $500^{\circ} \mathrm{C}$. Y-axes are scaled in the same increments to show the differences in magnitudes. The set of $\mathrm{H}_{2}$ concentrations range from 200$10,000 \mathrm{ppm}$ and each set is repeated three times. spectrometer. With this setup, defects in optical 
more samples. Initial sensing data of the different films to the same $\mathrm{H}_{2}$ exposures are shown in Figure 3. The films will be further exposed to $\mathrm{H}_{2}, \mathrm{NO}_{2}$, and $\mathrm{CO}$ in an air background, $10 \%$, and $5 \% \mathrm{O}_{2}$ backgrounds and the details/results of this study are described in Section 3.4 of this report.

\subsection{Size Dependent Studies}
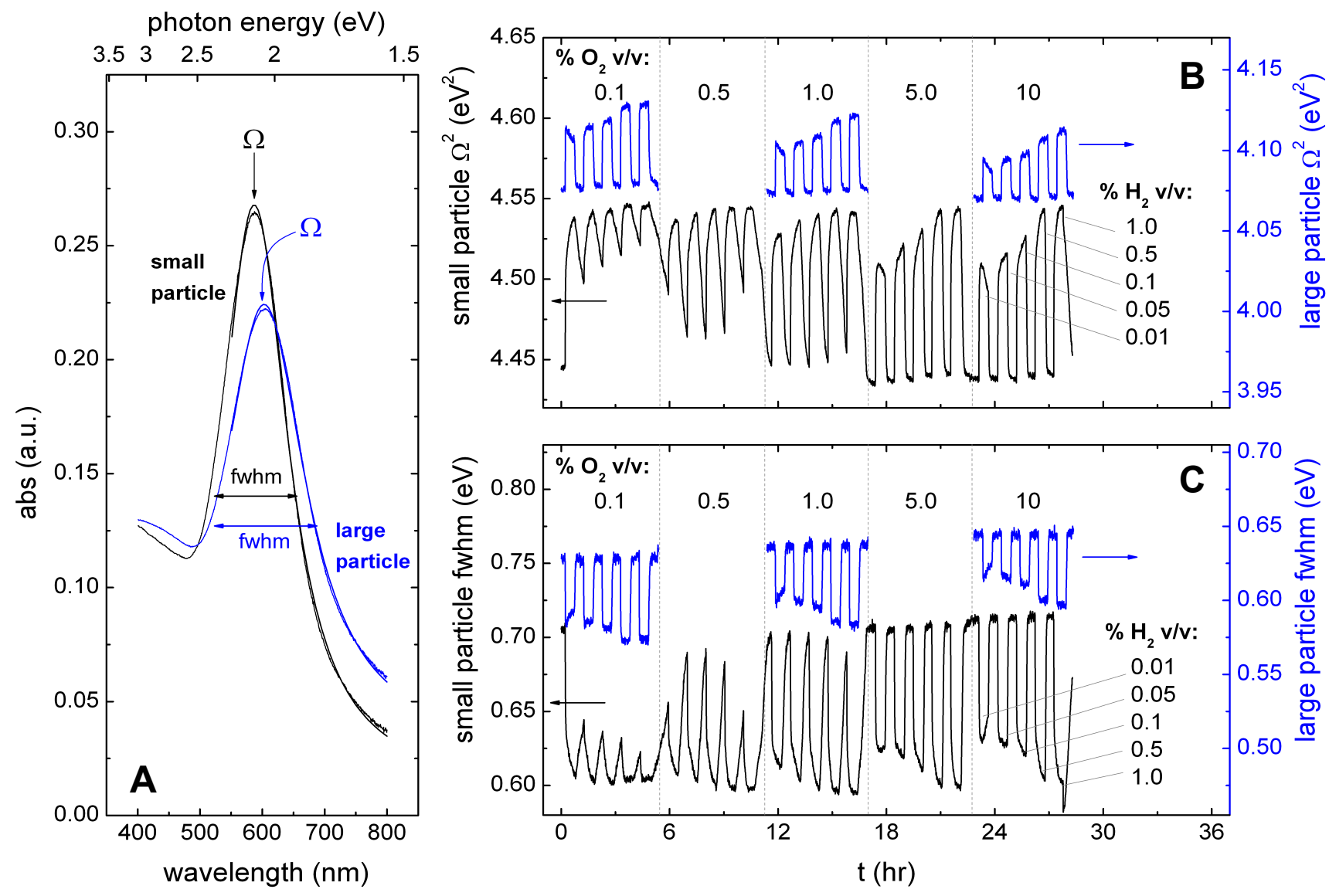

Figure 4. Room temperature absorption spectra and Lorentzian fits to the LSPR acquired for both the small and large particle Au-YSZ nanocomposite films (A). Also plotted are exposure data for one run of the three $500{ }^{\circ} \mathrm{C} \mathrm{H}_{2} / \mathrm{O}_{2}$ in $\mathrm{N}_{2}$ titration experiments after the absorption data has been fitted to a Lorentzian. Shown above are the square of the peak position (B) and the fwhm (C) as a function of time and exposure conditions that result from the fit. Gaps were placed in the large particle exposure data in (B) and (C), which was only collected at three background oxygen concentrations, so gas exposure concentration labels would correspond for all plotted data.

The development of sensor arrays with enhanced sensitivity and selectivity will depend on both the AuNP size as well as the metal oxide chemistry. The AuNP size was first investigated through the deposition and analysis of Au-YSZ films with AuNPs of two differing average particle sizes. Au-YSZ thin films were deposited using rf co-magnetron confocal physical vapor deposition (PVD) using deposition parameters similar to those published previously. ${ }^{1}$ After 
deposition, films are removed from the deposition chamber and were placed into an annealing chamber. The small and large particle films were then annealed at $\sim 900{ }^{\circ} \mathrm{C}$ for 2 and 3 hours, respectively, under $2000 \mathrm{sccm}$ flow of Ar at atmospheric pressure. TEM microscopy was used to approximate average grain sizes for the Au-YSZ nanocomposite thin films. The small particle film had an average particle size of $8 \mathrm{~nm}$ and the large particle film had an average particle size of $18 \mathrm{~nm}$.

$\mathrm{H}_{2} / \mathrm{O}_{2}$ titration experiments were completed to ascertain the dependence of the LSPR peak position and fwhm on the exposure environment for Au-YSZ nanocomposite films with AuNPs having average diameters of 8 and $18 \mathrm{~nm}$. Figure 4 shows example spectra and fits for both particle sized films, and exposure traces of the square of the peak position and the fwhm values generated from the Lorentzian fits to the experimental absorption spectra, traced as a function of time for the small ( $8 \mathrm{~nm}$ diameter) and large $(18 \mathrm{~nm}$ diameter) particle films for one run of titration exposure experiments. The fwhm data is shown in Figure 4 to illustrate that this parameter can be observed along with the peak position, and its corresponding variation as a function of gas exposure is in a direction which is consistent with previous work. ${ }^{2}$ Changes in the fwhm of the LSPR peak are an indication of a change in the plasmon dampening properties. Previous work has addressed the dampening properties with respect to inelastic scattering of plasmons by filled oxygen vacancies. ${ }^{2}$ Inspection of Figure 4 reveals several interesting characteristics, the most notable being the slow kinetics observed at low oxygen concentrations for the small particle film when compared to the large particle film. These slow kinetics are most evident in the recovery portion of the $\mathrm{H}_{2}$ exposure cycles for the 0.1 and $1.0 \% \mathrm{O}_{2}$ cycles of the small particle film. These recovery times are indeed prohibitively slow and within the given exposure cycle, the film has not reached its baseline equilibrium value prior to the next $\mathrm{H}_{2}$ exposure cycle. It has recently been shown by DeSouza et al. that grain boundaries in dense nanocrystalline YSZ inhibits oxygen ion diffusion, which likely also explains the slow optical response and recovery of the small particle nanocomposite film. ${ }^{3}$ Another qualitative observation of the data depicted in Figure 3 is that the magnitude of the change in the square of the LSPR peak position is greater for that of the small particles over that of the large particles, which agrees with the theoretical prediction that a smaller particle film will be more sensitive, especially in the range of low $\mathrm{H}_{2}$ exposure. A detailed theoretical model was developed for this experiment to understand how the equilibrium response of these films depends on both surface and in-film reactions as a function of particle size. Both the experimental and theoretical results are described in full in the 2010 Journal of Physical Chemistry C publication. ${ }^{4}$ In summary, while the small particle film appears to be more sensitive than the larger particle film, the PVD films have poor size control as the average particle size can vary by $10-20 \mathrm{~nm}$ in size. In order to achieve size control which is independent of the annealing temperature we are recently working on developing methods using electron beam lithographically patterned samples. 


\subsection{Kinetics Studies ${ }^{5}$}

In the size dependent titration studies, at low oxygen concentrations there appeared to be an emerging time dependence pattern that had not been previously observed. Upon removal of all the background oxygen using Matheson gas purification cartridges to purify the $99.999 \%$ pure gases, it became clear that the hydrogen gas was itself causing a change in the AuNP plasmon peak with a characteristic response time. These studies were continued and became a benchmark kinetics study on the $\mathrm{H}_{2}$ dissociative adsorption reaction on Au-YSZ as a function of $\mathrm{Au}$ nanoparticle (AuNP) diameter. These studies answered questions raised during the $\mathrm{H}_{2} / \mathrm{O}_{2}$ titration studies and display for the first time the use of the AuNP localized surface plasmon resonance band (LSPR) as

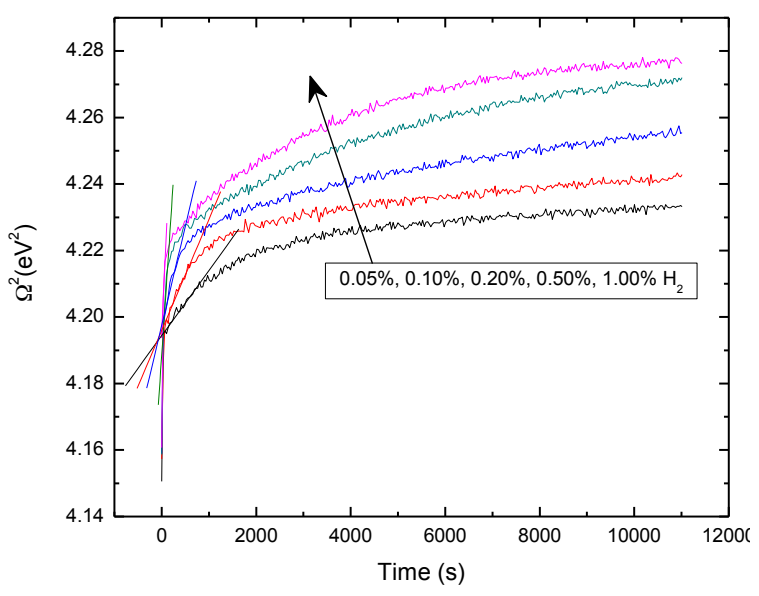

Figure 5: Linear fits to the start of the response for the small particle size. Data is overlapped to show $\mathrm{H}_{2}$ turned on at time equals zero for each concentration. The point of $\mathrm{H}_{2}$ off is not shown. a beacon for reaction kinetics under harsh environmental conditions. A study of the kinetics was done by performing three replicated experiments at each of the three temperatures; 300,400 , and $500^{\circ} \mathrm{C}$, and at each of the five concentrations; $0.05,0.10,0.20,0.50$, and $1.00 \% \mathrm{H}_{2}$ in a $\mathrm{N}_{2}$ background. In addition, both large $(\sim 25 \mathrm{~nm})$ and small $(\sim 15 \mathrm{~nm})$ particle sizes were exposed simultaneously in a parallel sensing configuration. The reaction rates were measured with a linear fit as shown with the overlaid data in Figure 5. Although a linear fit was the clear choice for the small particle films at $500^{\circ} \mathrm{C}$, at lower temperatures (such as in the figure) the response shows more of a decay towards saturation as opposed to a linear time relation. This has been addressed by making a linear fit to just the initial part of the response as shown in the figure. This type of analysis is the same as the classic method of initial rates type analysis and it is clear that the measured rate is dependent on the hydrogen concentration.

The measured rates seem to agree with a dissociative adsorption model when plotted against the partial pressure of hydrogen to the one-half power. Figure 6 shows this relation for the small particle film and a reasonably linear relation is seen for all three temperatures. Note that the reaction rates for the highest concentrations of $\mathrm{H}_{2}$ were not measureable for the small particle sample at 300 and $400^{\circ} \mathrm{C}$ so those data points were not included. The large particle film also showed a linear trend, and all five concentrations were measurable.

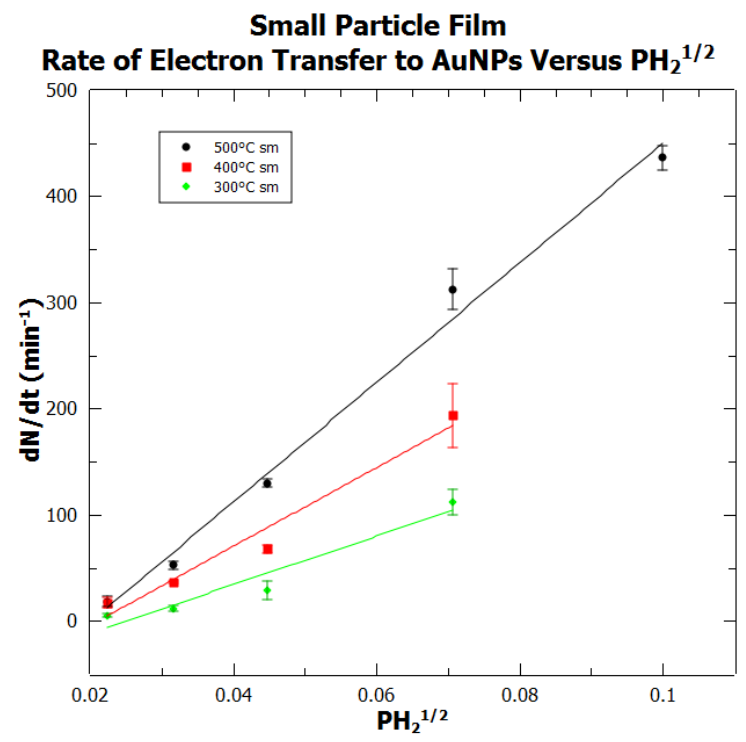

Figure 6: Half-order pressure dependence of the measured reaction rate at each of the $\mathrm{H}_{2}$ concentrations at 500,400 , and $300{ }^{\circ} \mathrm{C}$ for the large particle film. 


\subsection{Selectivity and Material Control: Au-Ceria Sensing Results ${ }^{6}$}

While we have shown evidence for modification of the Au-metal oxide's response to the target gases as a function of average gold particle size, we can also modify the metal oxide chemistry and determine how this affects the sensing characteristics. We collaborated with the EMSL facility of the Pacific Northwest National Laboratory, in particular Thevuthasan, Suntharampillai's group, in the deposition and characterization of AuNPs embedded in molecular beam epitaxially grown ceria thin films. The absorption spectrum for this sample is shown in Figure 7. There is a good fit between the sample data and the Lorentzian function that is used to extract the LSPR peak position.

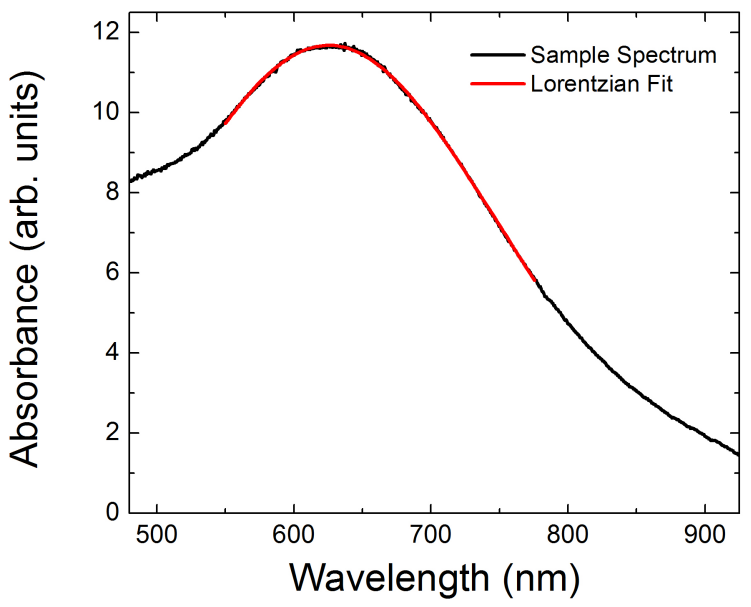

Figure 7: Example of the LSPR absorption spectrum and Lorentzian fit used to extract the peak position.

The square of the peak position is used as the sensing signal because, from the Drude model, it is proportional to the free electron density on the gold nanoparticles as shown in Equation 1:7

Equation 1: $\Omega^{2}=\frac{\mathrm{N} e^{2}}{\left(1+2 \varepsilon_{m}\right) \mathrm{m}_{e} \varepsilon_{0}}$

Where $\Omega$ is the surface plasmon resonant frequency, $\mathrm{N}$ is the density of conduction electrons, $e$ is the elementary charge, $\varepsilon_{\mathrm{m}}$ is the dielectric function of the matrix, $\mathrm{m}_{e}$ is the electron mass, and $\varepsilon_{0}$ is the permittivity of free space. As a result of earlier work that modeled the sensing response on the basis of a charge exchange mechanism, it is believed that changes in the free electron density can play a significant role in the LSPR peak shifts. By monitoring the LSPR peak position-squared as a function of time during gas exposures, the exposure plots are generated as shown by the example given in Figure 8. 

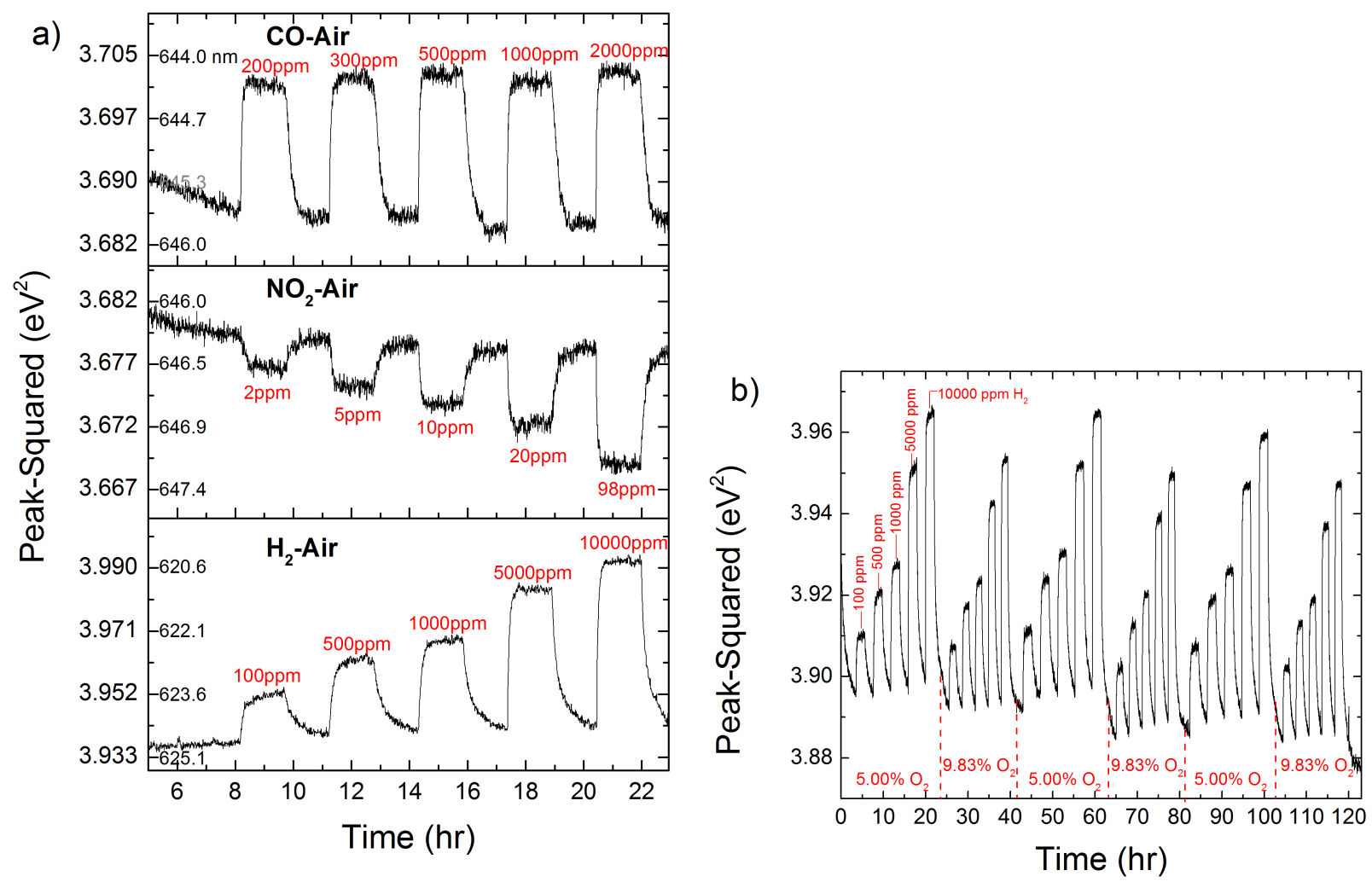

Figure 8: The square of the LSPR peak position during exposures at $500^{\circ} \mathrm{C}$. a) Analyte on-off cycles with the concentrations shown using air as the carrier gas. The y-axis shows the peak position squared in units of $\mathrm{eV}^{2}$, with the associated values in nanometers also listed as a reference. b) Example of a long-run exposure test for $\mathrm{H}_{2}$ in two different background $\mathrm{O}_{2}$ levels.

In each of the three separate experimental runs shown in Figure 8, the LSPR peak positionsquared is plotted while the analyte gas off-on cycles are repeated for increasing concentrations in an air background at $500^{\circ} \mathrm{C}$. The most obvious result is that $\mathrm{NO}_{2}$ exposure causes the peak position to red-shift (decrease in energy) while $\mathrm{CO}$ and $\mathrm{H}_{2}$ cause a blue-shift. This is the same type of response seen for Au-YSZ films and it would be expected to be the same if the response is due to the process of $\mathrm{O}^{2-}$ incorporation or withdrawal, respectively, from the film. Figure 8(b) shows an example of a long-run (120 hours) exposure test for $\mathrm{H}_{2}$ in two different $\mathrm{O}_{2}$ levels. It is apparent from this data set that this material set has stable and reliable sensing characteristics. It is noteworthy to realize that not only is the material stable for long periods of time in a harsh environment, but the film is easily able to detect $\mathrm{NO}_{2}$ at $2 \mathrm{ppm}$ concentrations in the presence of an air carrier gas. Such reliability and sensitivity is further evidence of the promise of this technique for continued development as a harsh environment compatible chemical sensor.

\section{Selectivity: Multivariate Analysis}

At issue with all of our previous studies is that reducing gases, $\mathrm{H}_{2}, \mathrm{CO}$ or hydrocarbons cause a blue-shift in the peak position when the analyte gas is turned on, which will certainly cause problems with selectivity as each of them will look nearly identical with respect to their general shift in the plasmon band upon exposure. Moreso, oxidizing gases, $\mathrm{O}_{2}$ or $\mathrm{NO}_{2}$ both cause a red shift upon exposure and will be equally difficult to selectively detect. More specifically to the 
current results, with the data shown from Figure 8, peak shifts resulting from the lowest concentration of $\mathrm{H}_{2}$ are within the whole range of peak shifts for all of the $\mathrm{CO}$ exposures. Therefore, if only the peak position is monitored, there would be no distinction between $\mathrm{H}_{2}$ and $\mathrm{CO}$ target gases within this overlapping region. However, there is a full absorption spectrum from which additional information may be extracted by using hyperspectral multivariate analysis techniques in order to find some distinction between the two analytes. PCA is a common method for this purpose, and it is often used for sensor analysis to reduce the dimensionality of multiple highly-correlated measurements. Usually these measurements come from an array of different sensing elements, but it is also possible to use multiple measurements from a single sensing film as an input to PCA. The raw data consist of $\mathrm{X}$ number of data points sitting in Ndimensional space, where $\mathrm{N}$ is the number of measured variables recorded for $\mathrm{X}$ observations. PCA performs a change of basis by defining an ordered set of orthogonal vectors such that the first points in the direction of maximum variance in the data, the second points in the direction of maximum variance while still being orthogonal to the first, and so on. These new axes are the principal component (PC) axes of the data set. Mathematically, the PCs are the eigenvectors of the covariance matrix and they are ranked according to the eigenvalues. Further details on this analysis method can be found elsewhere. ${ }^{8,9}$ Dimensional reduction can be performed by projecting the data onto a subspace consisting of just the first few PCs, which describe most of the variance in the data. When the data is plotted in PC space (known as the PC scores plot) similar types of sensor responses tend to form clusters. These clusters may, for example, represent exposure to different analytes and would indicate a unique sensing response to each if the clusters do not overlap.

For the present study, the change in absorption intensity upon gas exposure was recorded for every integer wavelength in the absorption spectrum between 585 and $750 \mathrm{~nm}$. This was found by subtracting the Lorentzian fit of the data in the gas-off state from the Lorentzian fit in the gason state, and resulted in a total of 166 measured variables. This was done for 45 observations (the result of 3 analytes times 5 concentrations times 3 oxygen background levels) and the measured variables were autoscaled prior to performing PCA in order to have mean-centered data with unit variance. The singular value decomposition (SVD) method was used with the Python programming language to generate the PC vectors and the PC scores plots shown in Figure 9. The 45 original observations are seen to cluster into three groups corresponding to the three different analytes, which qualitatively indicates a unique sensing response to each. In other words, the clusters allow for analyte classification in the concentration ranges tested. While there is no overlap between $\mathrm{H}_{2}$ and $\mathrm{CO}$ for the concentrations shown in Figure 9(a), a greater distinction between these two is seen on the PC3 vs PC2 axes in Figure 9(b), which clearly shows that the $\mathrm{CO}$ response can be identified over $\mathrm{H}_{2}$ and $\mathrm{NO}_{2}$. Thus, the PCA results show evidence of selective response for separate exposures and provide a basis on which to work towards selective sensing in gas mixtures. 

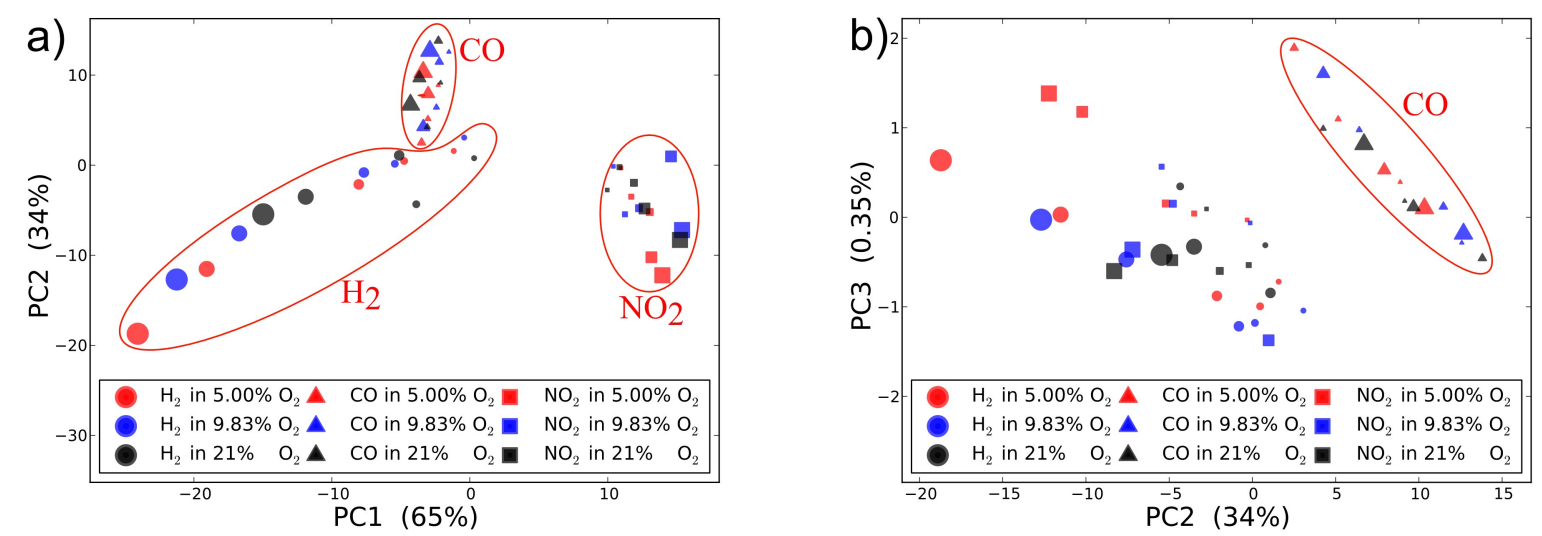

Figure 9: PC scores plots showing the data projected onto the PC axes. a) PC2 vs PC1, and b) PC 3 vs. PC 2 with the percent of total variance described by each PC also listed on the axes. Nonoverlapping clusters indicate a unique response to each of the three analytes. Data marker size increases with increasing concentration.

\subsection{Array Data: PCA Analysis ${ }^{10}$}

In general, selectivity would not be obtained under realistic conditions with only one sensing element. Therefore, we have deposited and analyzed results from a sensor array using three elements. Element one is a typical Au-YSZ sensing film used from our previous studies. Element two is a $\mathrm{Au}-\mathrm{TiO}_{2}$ film deposited and processed in an identical fashion as the Au-YSZ film except a titania PVD target was used during deposition. The third element is the $\mathrm{Au}-\mathrm{CeO}_{2}$ film obtained from PNNL and is described above. Data analysis from that acquired for the sensor array containing the $\mathrm{Au}-\mathrm{CeO}_{2}$ sample element as well as elements consisting of $\mathrm{Au}-\mathrm{YSZ}$, Au$\mathrm{TiO}_{2}$, and a sample with surface gold particles on $\mathrm{TiO}_{2}$ have been completed, a sample of which is shown in Figure 3 above. Examination of this data shows that as a function of time each of these sensor elements shows stability and a reliable response, except for the surface $\mathrm{Au}-\mathrm{TiO}_{2}$ element. A series of calibration curves which detail the plasmon peak position vs. target gas concentration and FWHM vs. target gas concentration have been plotted and a comparison between the individual sensing elements has noted that while the $\mathrm{Au}-\mathrm{YSZ}, \mathrm{Au}-\mathrm{TiO}_{2}, \mathrm{Au}-\mathrm{CeO}_{2}$ samples all perform well, a determination of the selectivity characteristics is problematic as both $\mathrm{H}_{2}$ and $\mathrm{CO}$ respond in a similar manner.

PCA analysis of the data for both the individual sensing elements as well as that from the sensing array in its entirety have been completed and will be used to demonstrate selectivity characteristics in an upcoming manuscript. A sample of this data can be seen in Figure 10. To generate this plot the entire surface plasmon absorption spectrum obtained with the target gas one was subtracted from the plasmon spectrum when the target gas was off. In this fashion a difference spectrum was obtained and this was used as the data input for the PCA algorithm. Given that we used 3 target gases, $\mathrm{CO}, \mathrm{H}_{2}$ and $\mathrm{NO}_{2}$, each with 5 concentrations in 3 oxygen concentrations, these 45 observations were used against 630 variables (wavelengths spanning across the absorption spectrum) for the 3 sensing elements, for a total of 1890 variables as the total input into the PCA algorithm. The output from this analysis is best viewed as a principal components plot as shown in Figure 10. It is clear that the target gases are clustered well and 
with sufficient separation from one another, thus providing evidence for the selectivity characteristics of this sensing array.

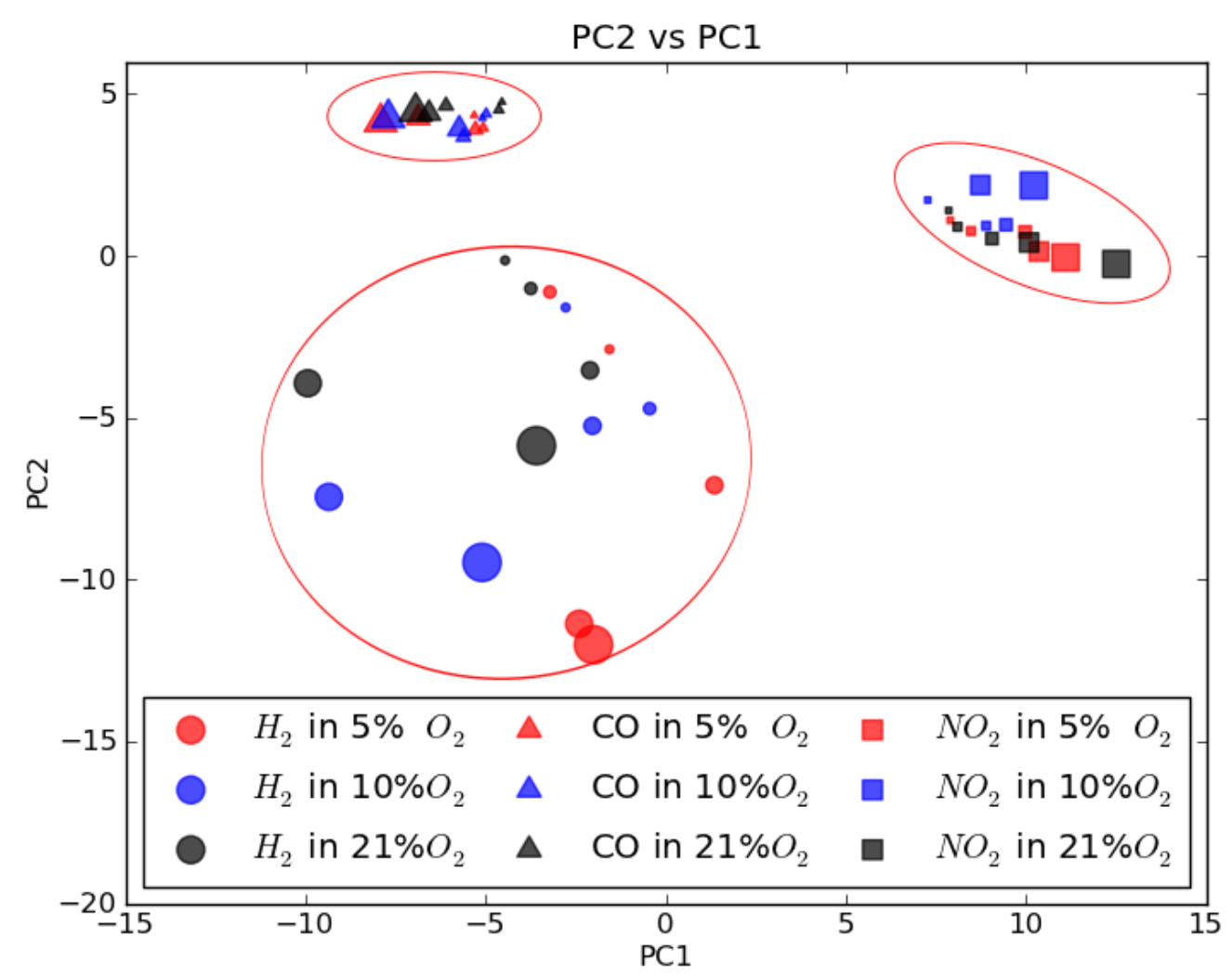

Figure 10: PCA plot for data obtained using a 3 element sensing array.

\subsection{Material Control: Embedded Gold Nanorods}

The development of selective chemical sensors is not only dependent on changes in the chemical composition of the metal oxide material, but also the size and shape of the Au nanoparticle itself. Haruta has shown that the catalytic oxidation of $\mathrm{CO}$ on supported gold nanoparticles is strongly dependent on the size of the gold nanoparticle. ${ }^{11}$ A complication with our typical methods is that our PVD deposited Au-metal oxide films have poor size control, with some size distributions being as large as the average particle diameter(i.e. $20 \mathrm{~nm}+/-20 \mathrm{~nm}$ ). To obtain samples with a narrower size distribution we have investigated the use of electron beam lithographically patterned arrays of Au nanoparticles. While one can print nearly any shape that is required using e-beam lithography, for the purposes of these studies the nanoparticles need to be stable at elevated temperatures. Our nanoparticle stability studies have shown that with a step-wise annealing process nanoparticle arrays with as thin as a $30 \mathrm{~nm}$ overcoat of the metal oxide can be stabilized even up to an annealing temperature of $800^{\circ} \mathrm{C}$. Thus creating nanoparticle arrays for stable operation at $500^{\circ} \mathrm{C}$ is clearly possible.

A typical example of a sample consists of Au nanorods $(44 \times 100 \mathrm{~nm})$ encapsulated in a $65 \mathrm{~nm}$ underlayer of YSZ and a $15 \mathrm{~nm}$ overlayer of YSZ. For a schematic of this structure see Figure 
11A. This sample was annealed stepwise to $600^{\circ} \mathrm{C}$ and there was no change in the surface as seen in the ESEM images acquired after the annealing process. The radial plasmon mode was observed at $620 \mathrm{~nm}$ while the longitudinal mode was observed at $800 \mathrm{~nm}$ in the spectrum, Figure 11C). As further evidence of the nanorod stability, environmental scanning electron microscopy was performed on these samples and from the images in Figure 11B it is clear that even after annealing the rods have maintained their shape. This is a critical aspect of this program. At the end of this program we were nearly done completing the stability study and had also begun gas exposure studies, however it is expected that by being able to tune the size and shape of the gold nanorod as well as the metal oxide chemistry, new elements for selective chemical sensor arrays will be possible.
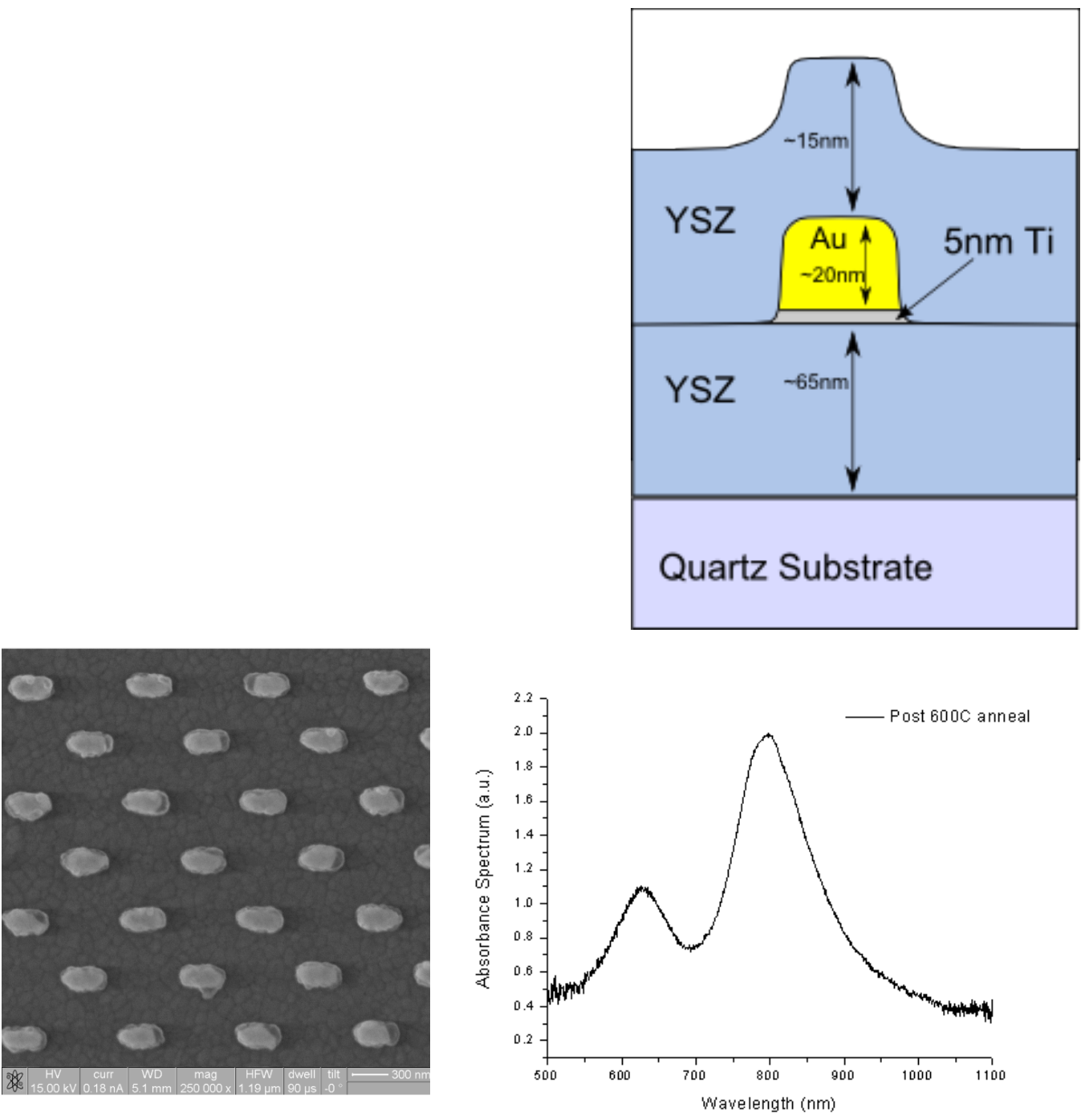

Figure 11: A) Schematic outlining structure of YSZ embedded Au nanorods, B)ESEM images of nanorods after annealing to $600^{\circ} \mathrm{C}, \mathrm{C}$ ) Plasmon absorption spectrum of nanorods from B) 


\subsection{Conclusions}

The work done in this program between January 2009 and December 2011 has continued to improve the viability of plasmonics based chemical sensing methods for harsh environment conditions. Prior to the start of the program, the concepts were sound, however more work was needed to be completed to prove the viability of a reliable sensing array methodology for enhanced selective responses to the target gases. This program had the following 5 research initiatives: 1)Parallel Materials Deposition, 2)Parallel Sensing Studies, 3) Sensing Characterization, 4)Materials Optimization, and 5)Selectivity Studies. Each of the initiatives were pursued in this program and with the development of plasmonic sensing array measurement techniques as well as the demonstration of how a change in metal oxide chemistry coupled with gold metal nanoparticle dopants for use in a plasmonic based sensor has shown great promise for the detection of a variety of target gases as well as operation environments. The variety of metal oxides that have been used as the embedding material for these plasmonic based sensors includes YSZ, $\mathrm{TiO}_{2}$ and $\mathrm{CeO}_{2}$. The addition of the oxygen ion conduction properties enhances the interfacial reaction characteristics as oxygen anions can more readily populate the entire metal oxide thin film due to their enhanced diffusion properties. This allows for sensing events to include both surface and bulk reactions. It is clear that the studies outlined above hold much promise for the use of optical based transduction methods in chemical sensors. However, as with a majority of sensing research work is still needed to continue to improve detection limits, reliability, and selectivity both with respect to the variety of target gases but also the background gases which are characteristic of the particular sensing application. Achievement of selectivity will most likely require the use of sensing arrays with a detailed statistical analysis to ensure the proper interpretation of the sensing "fingerprint" across the array elements. For this body of work the use of PCA methods has demonstrated that the target gases of interest have a unique response on the sensing arrays. Future work will be aimed at improving these arrays as well as improving the statistical analysis algorithms to characterize "unknown" target gases (after proper calibration and training of the array) both individually as well as within a gas mixture. It is this last point, gas mixtures, that will be challenging, however with continued optimization of the sensing methods, arrays (materials development) as well as analysis algorithms it appears that a plasmonics based method is promising to tackle this harsh environment sensing challenge.

\subsection{Index of acronyms}

PCA: principal component analysis

PC: principal components

YSZ: yttria-stabilized zirconia

XRD: $x$-ray diffraction

TEM: transmission electron microscopy

FWHM: full width half maximum

PNNL: Pacific Northwest National Laboratory

SVD: singular value decomposition

vs: versus

LSPR: localized surface plasmon resonance

AuNP: gold nanoparticle

EMSL: environmental and molecular sciences laboratory

PVD: physical vapor deposition

CCD: charge coupled device 
Rf: radio frequency

sccm: standard cubic centimeters per minute

$\mathrm{nm}$ : nanometers 


\subsection{References:}

${ }_{2}^{1}$ Rogers, P. H.; Sirinakis, G.; Carpenter, M. A. Direct Observations of Electrochemical

2 Rogers, P. H., Carpenter, M. A., Defect State Dampening of Surface Plasmons in Au-YSZ Nanocomposites, Proc. SPIE, 2009, 7395, 739519/1-19

${ }^{3}$ De Souza, R. A.; Pietrowski, M. J.; Anselmi-Tamburini, U.; Kim, S.; Munir, Z. A.; Martin, M. Oxygen Diffusion in Nanocrystalline Yttria-stabilized Zirconia: The Effect of Grain Boundaries Phys. Chem. Chem. Phys. 2008, 10, 2067-2072.

${ }^{4}$ Phillip H. Rogers and Michael A. Carpenter, Particle Size Sensitivity Dependence of Nanocomposites for Plasmonic-Based All-Optical Sensing Applications, J. Phys. Chem. C 2010, 114, 11033-11039

${ }^{5}$ Joy, N. A., Settens, C. M., Matyi, R J., Carpenter, M. A., "Plasmonic Based Kinetic Analysis of Hydrogen Reactions within Au-YSZ Nanocomposites", Journal of Physical Chemistry C 115, 6283-6289, (2011).

${ }^{6}$ Nicholas A. Joy, Manjula I. Nandasiri, Phillip H. Rogers, Weilin Jiang, Tamas Varga, Satyanarayana V N T Kuchibhatla, Suntharampillai Thevuthasan, Michael A. Carpenter, Selective Plasmonic Gas Sensing: $\mathrm{H}_{2}, \mathrm{NO}_{2}$, and $\mathrm{CO}$ Hyperspectral Discrimination by a Single $\mathrm{Au}$-CeO $\mathrm{O}_{2}$ Nanocomposite Film, Analyt. Chem., 84, 5025 (2012)

${ }^{7}$ Kreibig, U.; Vollmer, M. Optical Properties of Metal Clusters; Springer: Berlin, 1995.

8 Jackson, J. E. A User's Guide to Principal Components; Wiley-IEEE, 2003.

9 Jolliffe, I. T. Principal Component Analysis; Springer, 2002.

${ }^{10}$ Nicholas A. Joy, Manjula I. Nandasiri, Phillip H. Rogers, Satyanarayana V N T Kuchibhatla, Suntharampillai Thevuthasan, Michael A. Carpenter, Selective Plasmonic Gas Sensing: $\mathrm{H}_{2}, \mathrm{NO}_{2}$,

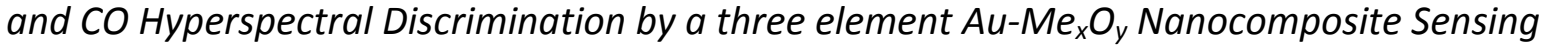
Array, In preparation (June 2012).

${ }^{11}$ M. Haruta, "Size- and support-dependency in the catalysis of gold," Catalysis Today, vol. 36, no. 1, pp. 153-166, Apr. 1997. 